\title{
Relação do linfedema e da fisioterapia na qualidade de vida de pacientes com câncer de mama
}

Impact of lymphedema and

physiotherapy in quality of life

FisiSenectus . Unochapecó Ano 7, n. 2 - Jul/Dez. 2019 of breast cancer patients p. $67-79$

Ligiane Martins de Souza Cordeiro. ligims.fisio@gmail.com

Fisioterapeuta. Egressa do Programa de Mestrado em Biociências e Saúde da Universidade do Oeste de Santa Catarina (Unoesc).

Talitta Padilha Machado. talittapm@yahoo.com.br

Fisioterapeuta. Egressa do Programa de Mestrado em Biociências e Saúde da Universidade do Oeste de Santa Catarina (Unoesc).

Carina Rossoni. carina.rossoni@unoesc.edu.br

Nutricionista. Doutora em Medicina e Ciências da Saúde pela Pontifícia Universidade Católica do Rio Grande do Sul. Professora do Programa de Pós-Graduação Stricto Sensu em Biociências e Saúde da Universidade do Oeste de Santa Catarina (Unoesc).

Marcelo Taglietti. marcelotaglietti@gmail.com

Fisioterapeuta. Doutor em Educação Física pela Universidade Estadual de Londrina. Professor do Centro Universitário Assis Gurgacz.

Marcelo Carlos Bortoluzzi. mbortoluzzi@gmail.com

Dentista. Doutor em Odontologia (Estomatologia Clínica) pela Pontifícia Universidade Católica do Rio Grande do Sul.

Antuani Rafael Baptistella. antuani.baptistella@unoesc.edu.br

Fisioterapeuta. Doutor em Oncologia - A.C. Camargo Cancer Center. Professor do Programa de Pós-

Graduação Stricto Sensu em Biociências e Saúde da Universidade do Oeste de Santa Catarina (Unoesc).

\section{Resumo}

Introdução: o câncer de mama é a neoplasia mais comum em mulheres no mundo, sendo que a morbidade e mortalidade resultantes dessa condição tornaram-se um problema de saúde. Uma das complicações decorrentes do tratamento do câncer de mama é o linfedema, condição que traz limitações funcionais e diminuição da qualidade de vida (QV) das pacientes. O tratamento fisioterápico é uma das abordagens mais utilizadas para prevenção e tratamento do linfedema. Objetivo: avaliar o impacto do linfedema e do tratamento fisioterápico em pacientes com câncer de mama. Métodos: foi avaliada a QV de mulheres diagnosticadas com câncer de mama na região Meio-Oeste de Santa Catarina através do instrumento genérico WHOQOL-BREF e do EORTC QLQ-BR23, questionário específico para câncer de mama. Com o objetivo de identificar fatores que interfiram na QV, foi analisada a presença ou ausência de linfedema e se essas pacientes realizaram fisioterapia para prevenir ou tratar possíveis complicações do tratamento. Resultados: das 135 
pacientes avaliadas, 33,3\% delas apresentavam linfedema no membro superior homolateral à cirurgia no momento da coleta, porém a presença de linfedema não afetou negativamente a QV das pacientes. Apenas $34 \%$ das pacientes realizaram fisioterapia em algum momento do tratamento, sendo que a realização desta não foi determinante para melhorar a QV. Conclusão: diante dos resultados encontrados, conclui-se que o diagnóstico de linfedema parece não afetar negativamente a QV geral das mulheres com câncer de mama avaliadas neste estudo, e que o tratamento fisioterápico não influenciou positivamente na qualidade de vida dessas pacientes. Ressalta-se a necessidade de novos estudos que permitam um melhor entendimento das causas do baixo número de encaminhamento das pacientes com câncer de mama para o tratamento fisioterápico, bem como para uma melhor avaliação da importância e a eficácia desse tipo de tratamento na funcionalidade e QV dessas pacientes.

\title{
Palavras-chave
}

Câncer de mama; Linfedema; Fisioterapia; Oncofuncional; Qualidade de vida.

\begin{abstract}
Introduction: breast cancer is the most common neoplasia in women in the world, and the morbidity and mortality resulting from this condition have become a health problem. One of the resulting complication of breast cancer treatment is the Iymphedema, chronic condition and often incurable, that brings functional limitations and decreases patients' quality of life. The physiotherapeutic treatment is one of the most used approaches to prevent and treat the Iymphedema. Objective: evaluate the impact of lymphedema and physiotherapy in quality of life of breast cancer patients. Methods: it was evaluated the quality of life of women diagnosed with breast cancer in the Mid-West of Santa Catarina state through the generic instrument WHOQOL-BREF and EORTC QLQ-BR23, a specific questionnaire for breast cancer. Aiming to identify factors that interfere in quality of life, was analyzed the presence or absence of lymphedema and if these patients received physiotherapeutic follow-up to prevent or treat possible complications of the cancer treatment. Results: from 135 evaluated patients, 33.3\% of them presented lymphedema in the upper member homolateral to the surgery at the time of data collection; however, the presence of lymphedema did not affect negatively the patients' quality of life. Only 34\% of the patients received physiotherapeutic assistance in some period of the treatment, and interestingly, this assistance was not determinant to improve the patients' quality of life. Conclusion: with this results, we concluded that the lymphedema diagnosis did not affect negatively the quality of life of patients evaluated in this study, and the physiotherapeutic treatment did not influence positively the quality of life of these patients. Stands out the necessity of new studies that allow a better understanding of the causes of the low number of forwarded of breast cancer patients to the physiotherapist, as well better evaluate the importance and efficacy of this type of treatment on patients' functionality and quality of life.
\end{abstract}

\section{Keywords}

Breast Cancer; Lymphedema; Physiotherapy; Physiotherapy in Oncology; Quality of life.

\section{Introdução}

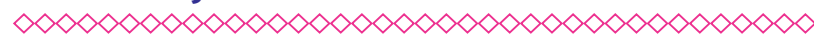

O câncer de mama é o segundo tipo de câncer mais frequente no mundo e o mais comum entre as mulheres. De acordo com a Organização Mundial da Saúde (OMS), que se baseia nos dados do documento World Cancer Report, do ano de 2014, publicado pela International Agency for Research on Cancer (IARC), o câncer se tornou um problema de saúde pública, principalmente entre os países em desenvolvimento, com uma estimativa de $20 \mathrm{mi}-$ Ihões de novos casos até $2025^{1}$. Os fatores de risco cada vez mais presentes e o crescente envelhecimento da população contribuem para o aumento do número de casos. 0 câncer de mama continua sendo a principal causa de morte por câncer entre as mulheres do mundo, com um número estimado de 1,7 milhão de casos e 521.900 em 2012². Segundo o Instituto Nacional do Câncer (INCA), para o Brasil, em 2019, são esperados 59.700 novos casos de câncer de mama³. 
A escolha do tratamento é realizada com base no estadiamento realizado pela classificação TNM, a qual avalia o tamanho do tumor $(\mathrm{T})$, o comprometimento linfonodal $(\mathrm{N})$ e a presença de metástase a distância (M). Além dessa classificação, o tratamento ainda leva em conta o subtipo molecular da doença. Atualmente, o tratamento para o câncer de mama envolve a combinação de diferentes terapias, incluindo cirurgia, radioterapia, hormonioterapia e quimioterapia ${ }^{4}$.

O tratamento cirúrgico para o câncer de mama compreende cirurgias conservadoras e não conservadoras, sendo que o esvaziamento axilar é considerado em casos em que há comprometimento linfonodal. 0 número de linfonodos ressecados está diretamente relacionado ao desenvolvimento do linfedema ${ }^{5,6}$. O linfedema é o acúmulo de líquido altamente proteico nos espaços intersticiais que leva ao edema do membro afetado devido ao bloqueio do sistema linfático, sistema responsável por conduzir a linfa pelo corpo, auxiliando no combate de infecções ${ }^{7}$. Outros fatores de risco para o desenvolvimento do linfedema são a idade avançada, o tamanho do tumor e a presença de infecção pós-operatória ${ }^{5-6,8-9}$. Além disso, a dor também pode ser um fator limitante para as pacientes, dificultando a realização de atividades do cotidiano e, de maneira geral, resultando em prejuízo na qualidade de vida das mulheres ${ }^{10,11}$.

O linfedema é uma doença crônica, progressiva e geralmente incurável, o que reforça a importância de intervenções precoces a fim de auxiliar essas mulheres a alcançar uma melhor qualidade de vida ${ }^{12}$. Além disso, causa um incômodo físico e emocional para as mulheres mastectomizadas, sendo que muitas delas experimentam depressão e ansiedade ${ }^{13}$.

É de fundamental importância conhecer os mecanismos envolvidos no desenvolvimento do linfedema no pós-operatório de câncer de mama para que possa haver uma efetiva prevenção e tratamento dessa complicação $0^{6}$. Um dos fatores que vêm contribuindo para uma menor incidência dessa complicação é o diagnóstico precoce e o progresso nas estratégias terapêuticas, em especial a técnica da biópsia do linfonodo sentinela ${ }^{14}$.

A fisioterapia em oncologia é uma especialidade recente e tem como metas preservar e restaurar a integridade cinético-funcional de órgãos e sistemas, assim como prevenir os distúrbios causados pelo tratamento oncológico, com foco nas sequelas próprias do tratamento, atuando de forma preventiva para minimizá-las ${ }^{15}$.

A fisioterapia tem como objetivo diminuir as complicações decorrentes de intervenções cirúrgicas nas pacientes mastectomizadas. 0 tratamento fisioterápico favorece a retomada das atividades diárias, melhorando, assim, a qualidade de vida através de condutas que reestabelecem os movimentos articulares e fortalecem a musculatura. A drenagem linfática tem o papel de agir sobre o linfedema, evitando possíveis complicações e restabelecendo a drenagem local normal no membro afetado. Porém, os melhores resultados aparecem quando há intervenção precoce do profissional fisioterapeuta no pós-operatório e, também, quando o tratamento tem início ainda na fase pré-operatória ${ }^{16}$.

0 linfedema pode ser uma das principais complicações do tratamento do câncer de mama, impactando de forma decisiva na qualidade de vida das pacientes ${ }^{2,13}$. Nesse sentido, uma adequada avaliação e acompanhamento fisioterapêutico são fundamentais para estabelecer um plano de tratamento e otimizar a evolução dessas pacientes.

Dessa forma, este trabalho apresenta o impacto do linfedema, a frequência do acompanhamento fisioterapêutico e a relação destes com a qualidade de vida das pacientes diagnosticadas com câncer de mama na região Meio-Oeste de Santa Catarina.

\section{Materiais e Métodos}

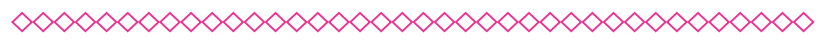

Trata-se de um estudo transversal, observacional, o qual foi aprovado pelo Comitê de Ética em Pesquisa Humana da Universidade do Oeste de Santa Catarina (Unoesc), sob parecer número 022539/2015.

\section{Amostra}

Foram incluídas todas as pacientes com diagnóstico médico de câncer de mama que estavam em fase de tratamento ou acompanhamento no 
setor de oncologia do Hospital Universitário Santa Terezinha (HUST), ou na Rede Feminina de Combate ao Câncer (RFCC) das cidades de Campos Novos, Capinzal e Joaçaba. Foram excluídas as pacientes que não apresentavam condições físicas ou nível de entendimento necessário para responder aos questionários da pesquisa.

\section{Coleta de dados}

Após assinatura do Termo de Consentimento Livre Esclarecido (TCLE), foram coletados os dados sociodemográficos (idade, estado civil, grau de instrução) e informações sobre a realização de acompanhamento fisioterapêutico ou não para tratar alguma complicação decorrente do tratamento para o câncer de mama, ambos através de um instrumento elaborado pelos autores. Em seguida, foi realizada a perimetria dos membros superiores para avaliação da presença de linfedema e avaliação da qualidade de vida das pacientes através de dois instrumentos: World Health Organization Quality of Life Bref (WHOQOL-BREF) (19), que se trata de um questionário genérico que possibilita a mensuração da qualidade de vida geral em pacientes oncológicos; e European Organization for Research and Treatment of Breast Cancer Quality of Life Questionnaire (EORTC QLQ-BR23), que, devido à sua configuração multifatorial, proporciona a mensuração da qualidade de vida e o impacto do tratamento especificamente para o câncer de mama ${ }^{17}$, ambos validados em língua portuguesa.

No instrumento WHOQOL, para cada aspecto da qualidade de vida expresso no questionário, o sujeito pôde apresentar sua resposta por meio de escores que variavam de 1 a 5 , sendo a pior condição no escore pontuada como 1 e a melhor, como 5. Os resultados dos domínios apresentam valores entre 4 e 20 , sendo piores os mais próximos de 4 e melhores os mais próximos de $20^{18}$. Já no EORTC QLQ-BR23, a pontuação das questões é dada em escalas tipo Likert, obtendo-se valores mínimos de 0 até valores máximos de 100 . 0 valor próximo de 100 corresponde a uma maior qualidade de vida, exceto nas escalas sintomáticas que avaliam a gravidade dos sintomas, ou seja, quanto maior o valor, menor será a qualidade de vida ${ }^{17}$.

O linfedema foi avaliado através da perimetria, considerando a diferença da medida da circunferência do membro superior do lado operado em relação ao membro contralateral. A medida da circunferência dos braços e antebraços foi realizada por meio de fita métrica simples, nos seguintes pontos: no braço, 7 e $15 \mathrm{~cm}$ acima do olecrano e, no antebraço, $10 \mathrm{~cm}$ abaixo do olecrano. Considerou-se a presença de linfedema quando houve diferença maior que $2,0 \mathrm{~cm}$ entre a circunferência do membro superior afetado em relação ao normal em, ao menos, uma das medidas ${ }^{8,19}$.

A análise estatística foi realizada no programa estatístico IBM SPSS ${ }^{\circledR}$, e os dados são apresentados em média \pm desvio padrão (DP). Os testes estatísticos utilizados foram o Kruskal-Wallis $\mathrm{H}$ e $\mathrm{O}$ Mann-Whitney $U$, sendo considerada diferença significante $p<0,05$.

\section{Resultados}

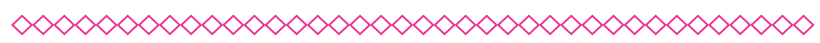

Foram incluídas 150 pacientes com diagnóstico de câncer de mama que realizaram tratamento no Hospital Universitário Santa Terezinha e estavam em acompanhamento na Rede Feminina de Combate ao Câncer nos municípios de Campos Novos, Capinzal e Joaçaba. Da amostra inicial, 5 (cinco) pacientes não conseguiram terminar de responder aos questionários, enquanto 10 (dez) foram eliminadas do estudo devido à falta de compreensão de questões; apenas uma paciente recusou-se a assinar o TCLE e a participar das entrevistas. Dessa forma, a amostra final do estudo foi de 135 pacientes.

A média de idade das pacientes foi de 53,8 anos, sendo que a mais jovem estava com 29 e a mais idosa, com 86 anos no momento das coletas. A maioria das mulheres era casada ou vivia com companheiro $(66,7 \%)$, enquanto $17 \%$ delas eram viúvas e $16,3 \%$ nunca casaram ou se encontravam separadas ou divorciadas. Em relação ao grau de instrução, 35,6\% das mulheres tinham o ensino médio completo, curso superior ou mais, enquanto $11,1 \%$ delas eram analfabetas (Tabela $\mathbf{1}$ ).

As pacientes deste estudo estavam em diferentes fases do tratamento no momento da coleta de dados para este estudo, sendo que a grande maioria já havia realizado a cirurgia para ressecção do tumor $(88,2 \%)$, enquanto apenas $11,8 \%$ das pacientes eram recém-diagnosticadas e 
estavam em tratamento neoadjuvante ou em fase pré-operatória. Entre as pacientes já operadas, a mastectomia foi o tipo de cirurgia mais comum, sendo realizada em $72,8 \%$ das mulheres operadas, enquanto a tumorectomia foi realizada em $27,2 \%$ das pacientes. A taxa de reconstrução de mama foi de $23,7 \%$, enquanto $76,3 \%$ das mulheres operadas não realizaram a reconstrução.

Das 135 pacientes avaliadas neste estudo, $33,3 \%$ delas apresentavam linfedema no membro superior homolateral à cirurgia no momento da coleta, enquanto $66,7 \%$ das pacientes não apresentaram um aumento da circunferência do membro superior homolateral à cirurgia maior que $2 \mathrm{~cm}$ quando comparado ao membro contralateral.

Quando se avaliou a qualidade de vida das pacientes através do instrumento WHOQOL-BREF, foi observado que o linfedema parece não afetar negativamente a qualidade de vida geral das pacientes com câncer de mama deste estudo, pois, em todos os domínios, as pacientes com linfedema apresentaram melhores escores de qualidade de vida, sendo que, no domínio psicológico e no de ambiente, as diferenças foram estatisticamente significativas ( $p=0,006$ e $p=0,019$, respectivamente) (Tabela 2).

Quando a qualidade de vida foi avaliada pelo EORTC QLQ-BR23, não foi observada nenhuma diferença estatística entre pacientes com e sem linfedema, em nenhum dos domínios do questionário, porém as mulheres com linfedema apresentaram piores médias nos domínios função sexual e prazer sexual, o que sugere uma tendência a menor qualidade de vida destas pacientes (Tabela 3).

Neste estudo, apenas 34\% das pacientes receberam atendimento fisioterapêutico em algum momento do tratamento para o câncer de mama ou no período de acompanhamento, 57,8\% não receberam nenhum tipo de assistência fisioterapêutica, enquanto $8,2 \%$ estavam em um período de recém-diagnóstico, o que sugere que não houve tempo para um possível encaminhamento à fisioterapia.

Ao comparar a qualidade de vida das pacientes que realizaram fisioterapia com a das pacientes que não realizaram, utilizando o questionário WHOQOL-BREF, não foi observada nenhuma diferença estatisticamente significante, porém, em todos os domínios, quem não realizou tratamento fisioterápico apresentou melhores escores de qualidade de vida (Tabela 4).

A avaliação de qualidade de vida, através no instrumento EORTC QLQ-BR23, entre pacientes que realizaram e não realizaram fisioterapia, mostrou uma diferença significativa $(p=0,044)$ no domínio que trata dos sintomas do braço, no qual quem realizou alguma intervenção fisioterapêutica teve maiores médias $(34,54 \pm 22,61)$, o que sugere uma pior qualidade de vida em relação a quem não recebeu nenhum tipo de atendimento $(26,35 \pm 21,49)$. Nenhum dos outros domínios avaliados pelo instrumento mostrou diferença estatística entre as pacientes que realizaram ou não realizaram fisioterapia (Tabela 5 ).

\section{Discussão}

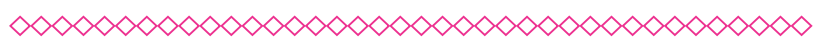

Uma das complicações pós-operatórias mais comuns nas mulheres com câncer de mama é o linfedema, que tem implicações diretas na qualidade de vida das pacientes ${ }^{2,17}$. Neste estudo, encontrou-se uma prevalência de linfedema em 33,3\% das pacientes avaliadas, considerando que, para avaliação da presença de linfedema, foram obtidas medidas de cada um dos membros superiores de maneira simétrica, e que a diferença de $2 \mathrm{~cm}$ a mais do lado homolateral à cirurgia foi considerada como presença de linfedema. No estudo de Meirelles e Gomide ${ }^{17}$, a incidência do linfedema variou de 6 a 43\%. Já a revisão realizada por Warren et al..$^{20}$ descreve que o linfedema pós-mastectomia ocorre de 24 a $49 \%$, pós-tumorectomia com dissecção axilar de 4 a $28 \%$, e de 5 a $34 \%$ após cirurgia e radioterapia.

Para as mulheres entrevistadas neste estudo, quando avaliadas pelo questionário específico (EORTC QLQ-BR23), o linfedema não teve influência na qualidade de vida, enquanto, no questionário genérico (WHOQOL-BREF), o linfedema foi responsável por escores maiores no domínio psicológico e ambiente, sugerindo melhor qualidade de vida nestas mulheres. Pesquisas anteriores descrevem resultados opostos aos encontrados neste estudo, pois apontam o linfedema como um problema 
estigmatizante que impacta de forma significativa a vida das mulheres que o desenvolvem, tanto física quanto emocional e socialmente ${ }^{21}$. Em um estudo realizado por $L_{a h o z}{ }^{22}$, utilizando o questionário SF-36, que avalia a qualidade de vida, também ficou claro que a presença do linfedema causa importantes alterações físicas, psicológicas e sociais na vida de mulheres que passaram por um câncer de mama.

0 resultado observado no presente estudo, em que o linfedema não interferiu negativamente na qualidade de vida das pacientes, pode ser justificado pelo critério utilizado para considerar a presença de linfedema, pois, apesar de ser definido na literatura um aumento em $2 \mathrm{~cm}$ na circunferência do membro, pode ainda ser assintomático e não influenciar de forma decisiva na funcionalidade e nas atividades de vida diária dessas mulheres, bem como na sua percepção corporal ${ }^{8,19}$.

A limitação física e funcional causada pela presença do linfedema, quando sintomático, interfere diretamente nas atividades diárias dessas mulheres, e a fisioterapia tem como um dos seus objetivos diminuir os efeitos limitantes dessa complicação ${ }^{13}$. A fisioterapia é uma estratégia terapêutica comumente utilizada em pacientes com câncer de mama que estão no pós-operatório ${ }^{22-25}$, principalmente com o objetivo de recuperação funcional desses pacientes ${ }^{15}$. Nos casos em que a complicação é o linfedema, o tratamento fisioterápico é altamente recomendado, pois, ao trazer benefícios funcionais às pacientes, influencia diretamente na qualidade de vida destas ${ }^{16,26,27}$.

Foi observado que as pacientes que realizaram fisioterapia obtiveram um resultado pior no domínio sintoma de braço do questionário específico, quando comparadas às mulheres que não realizaram fisioterapia. Tal resultado poderia ser explicado pelo fato de que as mulheres são mais frequentemente encaminhadas para o profissional fisioterapeuta quando já apresentam uma limitação, um sintoma ou uma complicação em decorrência do tratamento.

Os resultados de uma pesquisa desenvolvida por Silva et al. ${ }^{28}$ sobre a qualidade de vida pós-mastectomia e sua relação com o tratamento fisioterápico e o ganho de força muscular de membro superior apontaram que, quando encaminhadas de modo preventivo ao fisioterapeuta, as participantes não apresentavam alterações e que quanto mais precoce era o início da intervenção, melhores eram os resultados da reabilitação e a reinserção dessas mulheres em atividades cotidianas. Isso pode estar se refletindo em uma melhor qualidade de vida das pacientes que realizam o tratamento de forma preventiva. No estudo de Leites et al. ${ }^{29}$, a aplicação do protocolo de exercícios auxiliou na melhora dos parâmetros clínicos funcionais. Em outro trabalho realizado por Silva et al. ${ }^{29}$, a abordagem fisioterapêutica melhorou a amplitude de movimento, reduziu a dor, melhorou a função física e diminuiu os sintomas do braço, melhorando, assim, a qualidade de vida de mulheres após a cirurgia para câncer de mama.

A maioria das pacientes deste estudo não realizou fisioterapia para tratar ou prevenir o linfedema, ou mesmo outras limitações causadas pelo tratamento do câncer de mama. Esse dado deve ser considerado, pois retrata uma realidade na qual a maioria das pacientes com câncer de mama não é encaminhada ao profissional fisioterapeuta para receber um tratamento especializado que vise manter ou recuperar a função do membro suscetível ao linfedema, bem como tratar outras alterações funcionais decorrentes do tratamento que recebem. Novas pesquisas devem ser direcionadas para a ampliação do entendimento das causas do baixo número de encaminhamento das pacientes com câncer de mama para o tratamento fisioterápico, bem como para a comprovação da eficácia desse tipo de tratamento que tem por objetivos a manutenção da funcionalidade e o ganho de qualidade de vida para essas pacientes.

\section{Conclusão}

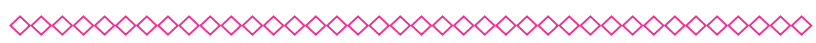

Em pacientes com diagnóstico de câncer de mama que estão em diferentes fases de tratamento, ou em acompanhamento em um hospital universitário ou na rede feminina de combate ao câncer, o diagnóstico de linfedema não afetou negativamente a qualidade de vida geral dessas mulheres. As pacientes com linfedema apresentaram melhores índices nos domínios psicológico e ambiente do questionário genérico, não havendo 
diferença significativa no questionário específico, apesar de apresentarem médias piores nos domínios função sexual e prazer sexual.

Além disso, neste estudo, o tratamento fisioterápico não influenciou positivamente na qualidade de vida das pacientes, já que a única diferença significante foi observada nas mulheres que realizaram fisioterapia e apresentaram mais sintomas de braço.

Outros estudos, com diferentes populações, devem ser realizados para se obter uma melhor compreensão da relação do linfedema e do tratamento fisioterápico com a qualidade de vida das pacientes com câncer de mama.

\section{Limitações do estudo}

Entre as principais limitações do estudo, destaca-se que, nesta análise, encontram-se todas as pacientes diagnosticadas com câncer de mama, e não apenas aquelas em fase pós-operatória, as quais normalmente apresentam linfedema como complicação cirúrgica. Aquelas que realizaram cirurgia não foram separadas pelo tipo de procedimento, sabendo-se que diferentes procedimentos cirúrgicos levam a diferentes riscos para o desenvolvimento de linfedema. Tais limitações se justificam pelo objetivo inicial do trabalho, que era identificar a prevalência geral de linfedema e o acompanhamento da fisioterapia e a relação destes com a qualidade de vida das pacientes com câncer de mama.

\section{Referências}

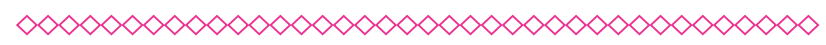

1. Ferlay J, Soerjomataram I, Dikshit R, Eser S, Mathers C, Rebelo M, et al. Cancer incidence and mortality worldwide: Sources, methods and major patterns in GLOBOCAN 2012. International Journal of Cancer. 2014.

2. Torre LA, Bray F, Siegel RL, Ferlay J, LortetTieulent J, Jemal A. Global Cancer Statistics, 2012. CA Cancer J Clin. 2015;65(2):87-108. doi: 10.3322/caac. 21262 .

3. INCA. Estimativa/2016 incidência de câncer no Brasil. Ministério da Saúde; 2015.
4. Nelson HD, et al. Screening for Breast Cancer: A Systematic Review to Update the 2009 U.S. Preventive Services Task Force Recommendation. Ann Intern Med. 2016;164(4):244-55. doi: 10.7326/M15-0969.

5. Rezende LFD, et al. Avaliação das compensações linfáticas no pós-operatório de câncer de mama com dissecção axilar através da linfocintilografia. J Vasc Bras. 2008;7(4):370-5. doi: 10.1590/S1677-54492008005000002.

6. Vieira RA, da Costa AM, de Souza JL, Coelho RR, de Oliveira CZ, Sarri AJ, et al. Risk Factors for Arm Lymphedema in a Cohort of Breast Cancer Patients Followed up for 10 Years. Breast Care (Basel). 2016 Feb;11(1):45-50. doi: 10.1159/000442489. Epub 2015 Dec 14.

7. Lawenda BD, Mondry TE, Johnstone PA. Lymphedema: a primer on the identification and management of a chronic condition in oncologic treatment. CA Cancer J Clin. 2009;59:8-24. doi:10.3322/caac.20001.

8. Bergmann A, Mattos IE, Koifman RJ. Diagnóstico do linfedema: análise dos métodos empregados na avaliação do membro superior após linfadenectomia axilar para tratamento do câncer de mama. Rev. bras. Cancerol. 2004;50(4):311-20.

9. Hopkins JO, Allred J, Hurria A, Jatoi A, Lafky JM², Cohen $\mathrm{H}$, et al. Lymphedema, musculoskeletal events and arm function in older patients receiving adjuvant chemotherapy for breast cancer Breast Cancer Res Treat. 2017 Aug 20. doi: 10.1007/s10549-017-4454-7.

10. Mendes IDS, et al. Correlação da dor e qualidade de vida de mulheres pós-tratamento cirúrgico de câncer de mama. Mundo Saúde. 2014;38(2):189-96. doi: 10.15343/01047809.20143802189196.

11. Stevens $P$, et al. Prevalence, characteristics, and impact of postmastectomy pain syndr ome: an investigation of women's experiences. Pain. 1995;61(1):61-8. doi: 10.1016/03043959(94)00162-8.

12. Panobianco MS, et al. Qualidade de vida de mulheres com linfedema após cirurgia por câncer 
de mama. Rev Rene. 2014;15(2):206-13. doi: 10.15253/2175-6783.2014000200004.

13. Calhoun C, Helzlsouer KJ, Gallicchio L. Racial diferences in depressive symptoms and selfrated health among breast câncer survivors on aromatase inhibitor therapy. J Psychosoc Oncol. 2015;33(3):263-77.

14. Soran A, D'angelo G, Begovic M, Ardic F, Harlak A, Samuel WH, et al. Breast cancerrelated lymphedema--what are the significant predictors and how they affect the severity of lymphedema? Breast Journal. 2006;12(6):536-43. doi: 10.1111/j.1524-4741.2006.00342.x.

15. Almeida MAB, Gutierrez GL, Marquez R. Qualidade de vida: definições e conceitos. São Paulo: Escola das Artes, Ciências e Humanidade EACH/USP; 2012.

16. Mori T, Lustman A, Katz-Leurer M. Selfmeasurement of upper extremity volume in women post-breast cancer: reliability and validity study. Physiother Theory Pract. 2015;31(4):283-7.

17. Meirelles MCCC, Gomide LB. Reabilitação da mulher submetida ao tratamento da neoplasia mamária: fase ambulatorial. Rio de Janeiro: Guanabara Koogan; 2011.

18. Fleck MPA, organizador. A avaliação da qualidade de vida: guia para profissionais da saúde. Porto Alegre: Artmed; 2008. doi: 10.1590/ S1413-81232009000600040.

19. Conde DM, Pinto-Neto AM, Freitas Júnior $\mathrm{R}$, Aldrighi JM. Qualidade de vida de mulheres com câncer de mama. Rev. bras. ginecol. Obste. 2006;28(3):195-204. doi: 10.1590/S010072032006000300010 .

20. Warren AG, Brorson H, Borud LJ, Slavin SA. Lymphedema: a comprehensive review. Ann Plast Surg. 2007;59(4):464-72. doi: 10.1097/01. sap.0000257149.42922.7e.

21. Assis MR, Marx AG, Magna LA, Ferrigno ISV. Morbidade tardia na função do membro superior e na qualidade de vida de mulheres pós-cirurgia do câncer de mama. Rev. bras. Fisioter. 2013;17(3):236-43. doi: 10.1590/S141335552012005000088 .
22. Lahoz MA, et al. Capacidade funcional e qualidade de vida em mulheres pós mastectomizadas. Rev. bras. Cancerol. 2010;56(4):426-30.

23. Vignes S, Porcher R, Arrault M, Dupuy A. Long-term management of breast cancer-related lymphedema after intensive decongestive physiotherapy. Breast Cancer Res Treat. 2007;101(3):285-90. doi:10.1007/s10549-0069297-6.

24. Sitzia J, Sobrido L, Harlow W. Manual lymphatic drainage compared with simple lymphatic drainage in the treatment of postmastectomy lymphoedema. Physiotherapy. 2002;88(2):99-107.

25. Uzkeser H, Karatay S, Erdemci B, Koc M, Senel K. Efficacy of manual lymphatic drainage and intermittent pneumatic compression pump use in the treatment of lymphedema after mastectomy: a randomized controlled trial. Breast Cancer. 2013. doi:10.1007/s12282-0130481-3.

26. Molski P, Kruczynski J, Molski A, Molski S. Manual lymphatic drainage improves the quality of life in patients with chronic venous disease: a randomized controlled trial. Arch Med Sci. 2013;9(3):452-5.

27. Guerero RM, Neves LMS, Guirro RRJ, Guirro ECO. Manual Lymphatic Drainage in Blood Circulation of Upper Limb With Lymphedema After Breast Cancer Surgery. J Manipulative Physiol Ther. 2017 May;40(4):246-9. doi: 10.1016/j.jmpt.2017.02.009. Epub 2017 Apr 8.

28. Silva $M D$, et al. Qualidade de vida e movimento do ombro no pós-operatório de câncer de mama: um enfoque da Fisioterapia. Rev. bras. Cancerol. 2013;59(3):419-26.

29. Leites GT, et al. Fisioterapia em oncologia mamária: qualidade de vida e evolução clínicofuncional. Ciência \& Saúde. 2010;3(1):14-21. doi: 10.15448/1983-652X.2010.1.6448. 


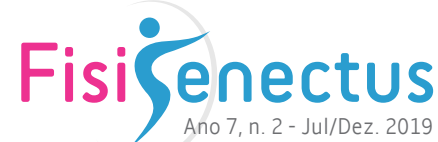

\section{Anexos}

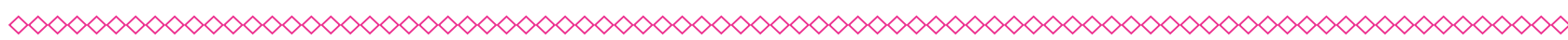

\begin{tabular}{|c|c|c|}
\hline Idade & $\mathrm{N}$ & $\%$ \\
\hline 29-49 anos & 47 & 34,8 \\
\hline $50-59$ anos & 51 & 37,8 \\
\hline $60-86$ anos & 37 & 27,4 \\
\hline \multicolumn{3}{|l|}{ Estado Civil } \\
\hline Nunca foi casado & 8 & 5,9 \\
\hline Casado ou vive com companheiro & 90 & 66,7 \\
\hline Separada ou divorciada & 14 & 10,4 \\
\hline Viúva & 23 & 17 \\
\hline \multicolumn{3}{|l|}{ Grau de Instrução } \\
\hline Analfabeto/Menos de um ano de instrução & 15 & 11,1 \\
\hline Elementar Incompleto & 40 & 29,6 \\
\hline Elementar Completo e Fundamental Incompleto & 22 & 16,3 \\
\hline $\begin{array}{l}\text { Fundamental Completo e Ensino Médio } \\
\text { Incompleto }\end{array}$ & 10 & 7,4 \\
\hline Ensino Médio Completo e Superior Incompleto & 34 & 25,2 \\
\hline Superior Completo ou mais & 14 & 10,4 \\
\hline
\end{tabular}

(clique para voltar ao texto) 
Tabela 2 - Linfedema e Qualidade de vida (WHOQOL-BREF)

\begin{tabular}{|c|c|c|c|c|c|c|c|c|}
\hline & \multicolumn{3}{|c|}{ Físico } & \multicolumn{2}{|c|}{ Psicológico } & \multicolumn{2}{|c|}{ Relações Sociais } & Ambiente \\
\hline & $\mathrm{n}$ & média ( $\pm \mathrm{DP}$ ) & $\mathrm{p}$ & média ( $\pm \mathrm{DP})$ & $\mathrm{p}$ & média $( \pm D P)$ & $\mathrm{p}$ & média ( $\pm \mathrm{DP})$ \\
\hline Sem Linfedema & 90 & $58,5( \pm 18,7)$ & $p=0,158$ & $64,3( \pm 17,7)$ & $p=0,006$ & $74,1( \pm 17,9)$ & $=0,061$ & $164,6( \pm 15,8) p=0,019$ \\
\hline Com Linfedema & 45 & $62,7( \pm 18,7)$ & & $72,9( \pm 13,6)$ & & $80,2( \pm 13,5)$ & & $71,0( \pm 12,9)$ \\
\hline
\end{tabular}

$\mathrm{n}$ = número absoluto de pacientes; $\mathrm{DP}$ = desvio padrão; significância estatística $\mathrm{p} \leq 0,05$.

O teste estatístico utilizado foi o de Mann-Whitney $\mathrm{U}$.

(clique para voltar ao texto) 


\begin{tabular}{|c|c|c|c|c|c|c|c|c|c|}
\hline & & $\begin{array}{l}\text { Imagem } \\
\text { Corporal }\end{array}$ & Função Sexual & Prazer Sexual & $\begin{array}{c}\text { Perspectivas } \\
\text { Futuras }\end{array}$ & $\begin{array}{c}\text { Efeitos Terapia } \\
\text { Sistêmica }\end{array}$ & $\begin{array}{l}\text { Sintomas da } \\
\text { Mama }\end{array}$ & $\begin{array}{c}\text { Sintomas do } \\
\text { Braço }\end{array}$ & $\begin{array}{c}\text { Chateada } \\
\text { Perda do } \\
\text { Cabelo }\end{array}$ \\
\hline & $\mathrm{n}$ & média ( $\pm D P$ ) & média ( $\pm D P$ ) & média ( $\pm D P$ ) & média ( $\pm D P$ ) & média ( $\pm D P$ ) & média ( $\pm D P)$ & média ( $\pm \mathrm{DP}$ ) & média ( $\pm \mathrm{DP})$ \\
\hline Sem Linfedema & 90 & $83,0( \pm 24,1)$ & $75,9( \pm 24,2)$ & $55,8( \pm 26,7)$ & $44,1( \pm 31,9)$ & $22,4( \pm 15,5)$ & $19,4( \pm 19,1)$ & $30,2( \pm 22,9)$ & $26,5( \pm 35,2)$ \\
\hline Com Linfedema & 45 & $89,6( \pm 16,4)$ & $75,2( \pm 26,0)$ & $53,3( \pm 28,9)$ & $51,9( \pm 35,2)$ & $19,9( \pm 14,5)$ & $19,1( \pm 13,0)$ & $24,9( \pm 19,8)$ & $11,1( \pm 20,6)$ \\
\hline Valor de $p$ & & 0,192 & 0,960 & 0,682 & 0,202 & 0,395 & 0,501 & 0,267 & 0,164 \\
\hline
\end{tabular}

$\mathrm{n}$ = número absoluto de pacientes; $\mathrm{DP}$ = desvio padrão; significância estatística $\mathrm{p} \leq 0,05$.

O teste estatístico utilizado foi o de Mann-Whitney U.

(clique para voltar ao texto) 


\begin{tabular}{|c|c|c|c|c|c|c|c|c|c|}
\hline & \multirow[b]{2}{*}{$\mathrm{n}$} & \multicolumn{2}{|c|}{ Físico } & \multicolumn{2}{|c|}{ Psicológico } & \multicolumn{2}{|c|}{ Relações Sociais } & \multicolumn{2}{|c|}{ Ambiente } \\
\hline & & média $( \pm D P)$ & $\mathrm{p}$ & média $( \pm D P)$ & $\mathrm{p}$ & média $( \pm \mathrm{DP})$ & $\mathrm{p}$ & média $( \pm D P)$ & $\mathrm{p}$ \\
\hline Realizou fisioterapia & 46 & $58,0( \pm 18,6)$ & $\mathrm{p}=0,371$ & $65,2( \pm 16,3)$ & $p=0,189$ & $74,2( \pm 18,4)$ & $p=0,661$ & $64,2( \pm 15,0)$ & $p=0,249$ \\
\hline Não realizou fisioterapia & 78 & $60,7( \pm 18,2)$ & & $68,6( \pm 17,3)$ & & $76,2( \pm 16,0)$ & & $67,5( \pm 15,6)$ & \\
\hline
\end{tabular}

$\mathrm{n}$ = número absoluto de pacientes; DP = desvio padrão; significância estatística $\mathrm{p} \leq 0,05$.

O teste estatístico utilizado foi o de Mann-Whitney U.

(clique para voltar ao texto) 


\begin{tabular}{|c|c|c|c|c|c|c|c|c|c|}
\hline & & $\begin{array}{l}\text { Imagem } \\
\text { Corporal }\end{array}$ & Função Sexual & Prazer Sexual & $\begin{array}{l}\text { Perspectivas } \\
\text { Futuras }\end{array}$ & $\begin{array}{l}\text { Efeitos Terapia } \\
\text { Sistêmica }\end{array}$ & $\begin{array}{l}\text { Sintomas da } \\
\text { Mama }\end{array}$ & $\begin{array}{c}\text { Sintomas do } \\
\text { Braço }\end{array}$ & $\begin{array}{c}\text { Chateada } \\
\text { Perda do } \\
\text { Cabelo }\end{array}$ \\
\hline & $\mathrm{n}$ & média ( $\pm D P$ ) & média ( $\pm D P$ ) & média ( $\pm \mathrm{DP})$ & média $( \pm D P)$ & média ( $\pm D P$ ) & média ( $\pm D P$ ) & média ( $\pm D P$ ) & média $( \pm D P)$ \\
\hline Realizou fisioterapia & 46 & $85,0( \pm 23,9)$ & $72,8( \pm 25,9)$ & $51,2( \pm 26,4)$ & $43,5( \pm 35,0)$ & $21,3( \pm 13,5)$ & $19,0( \pm 17,1)$ & $34,5( \pm 22,6)$ & $24,4( \pm 32,0)$ \\
\hline $\begin{array}{l}\text { Não realizou fisioterapia } \\
\text { Valor de } p\end{array}$ & 78 & $\begin{array}{l}84,1( \pm 21,8) \\
p=0,635\end{array}$ & $\begin{array}{l}75,9( \pm 24,7) \\
p=0,511\end{array}$ & $\begin{array}{l}56,9( \pm 29,1) \\
p=0,545\end{array}$ & $\begin{array}{l}46,2( \pm 32,3) \\
p=0,663\end{array}$ & $\begin{array}{l}21,6( \pm 16,8) \\
p=0,714\end{array}$ & $\begin{array}{l}20,8( \pm 17,8) \\
p=0,585\end{array}$ & $\begin{array}{l}26,4( \pm 21,5) \\
p=0,044\end{array}$ & $\begin{array}{l}25,3( \pm 34,4) \\
p=0,911\end{array}$ \\
\hline
\end{tabular}

$\mathrm{n}$ = número absoluto de pacientes; $\mathrm{DP}$ = desvio padrão; significância estatística $\mathrm{p} \leq 0,05$

O teste estatístico utilizado foi o de Mann-Whitney U.

(clique para voltar ao texto) 